\title{
Is the humeral stem useful in anatomic total shoulder arthroplasty?
}

\author{
Philippe Collin $^{1} \cdot$ Tetsu Matsukawa ${ }^{1,2} \cdot$ Pascal Boileau $^{3}$ - Ulrich Brunner ${ }^{4}$. \\ Gilles Walch ${ }^{5}$
}

Received: 1 March 2016 / Accepted: 11 December 2016/Published online: 3 January 2017

(C) The Author(s) 2017. This article is published with open access at Springerlink.com

\begin{abstract}
Purpose Traditionally and since Neer, the humeral side of shoulder arthroplasty consisted of a stemmed component but the real need for stem fixation in total shoulder arthroplasty (TSA) has barely been investigated. The current study evaluated the clinical and radiological outcomes with a stemless TSA.

Methods Forty-seven patients, 20 female and 27 male patients with an average age of 63 , were selected in four orthopaedic centres during a four year period, and implanted with a humeral head prosthesis with a three-fin design and titanium coating. Aetiologies were: primary osteoarthritis (29), fracture sequelae (12) and avascular osteonecrosis (6). Minimum follow-up was two years (range 24-51 months). The patients were evaluated with the Constant score (CS) and radiological exams.

Results Two patients had revision of the implants, one for persistent pain and one for secondary massive rotator cuff tear. At the final follow-up, the mean CS was 69, with an average gain of 36. All parameters improved with a foremost in pain
\end{abstract}

Philippe Collin

collin.ph@wanadoo.fr

1 Institut Locomoteur de l'Ouest CHP Saint Grégoire (vivalto Santé), 6 Boulevard de la Boutière, 35740 Saint Grégoire, France

2 Department of Orthopedic Surgery, Nagoya University School of Medicine, 65 Tsurumai-cho, Showa-ku, Nagoya 466-8550, Japan

3 Service de Chirurgie Orthopédique et Chirurgie du Sport, Institut Universitaire Locomoteur et du Sport, CHU de Nice Hôpital Pasteur, 230 Voie Romaine, 06000 Nice, France

4 Krankenhaus Agatharied GmbH, Norbert-Kerkel-Platz, 83734 Hausham, Germany

5 Hopital Privé J Mermoz (Générale de Santé), Centre orthopédique Santy, 24 avenue P Santy, 69008 Lyon, France relief. Mean satisfaction rate was $87 \%$. Average anterior active elevation was $131^{\circ}$ with a gain of 48 . Radiologic evaluations showed stable implants in all cases. However, 17 cases demonstrated radiolucent areas, particularly superior and lateral to the implant, which neither decreased nor increased with time. No revisions were related to humeral component loosening. Conclusions Stemless TSA provides the same results as compared to TSA with a humeral stem. We are still unsure as to the nature of the lucent zones and we are continuing our investigation to better understand this radiological phenomenon.

Keywords Total shoulder arthroplasty $\cdot$ Arthritis $\cdot$ Stem less

\section{Introduction}

Designs of early total shoulder arthroplasty (TSA) implants were based, in principle, on those of total hip arthroplasty (THA), with a cemented humeral stem. Since their introduction [1], four generations of stemmed TSA designs were developed, and demonstrated satisfactory clinical outcomes for reduction of pain and improvement of mobility. The most frequently reported complication, however, is loosening of the glenoid component [2-4]. The most common humeral complications are: intra-operative fracture, loosening, stress-shilding and periprosthetic fracture [4-6]. In addition, humeral complications may arise during stem removal for revision procedures. Nonetheless, the need for anchorage with a humeral stem was never demonstrated. Numerous authors reported the clinical outcomes of stemless shoulder arthroplasty designs with good results [7, 8]. The purpose of this study was to evaluate primary fixation as well as clinical and radiographic outcomes of a stemless TSA at a minimum follow-up of 24 months. Our hypothesis 
was that outcomes of stemless TSA would be equivalent to those of classic stemmed TSA designs.

\section{Materials and methods}

A prospective study protocol was prepared and approved by the institutional review board for this multicentre investigation prior to patient enrolment. Between July 2010 and July 2012 stemless TSA was performed on a continuous series of 47 patients, by four senior surgeons at four different centres. All patients gave informed consent for participation in this study and for use of their data for research and publication. Inclusion criteria were: patients operated for primary osteoarthritis, posttraumatic osteoarthritis and osteonecrosis. Exclusion criteria was revision arthroplasty. The patients included 20 men and 27 women with a mean age of 63 years (range 39-78) at the time of the index operation. The operated shoulder involved the dominant side in 30 patients. The indications for surgery were: primary osteoarthritis in 29 shoulders, post-traumatic osteoarthritis in 12 shoulders and aseptic osteonecrosis in six shoulders.

The operative technique was identical in all cases. The patients were seated in the beach-chair position under general anesthesia with an interscalene block. The surgical approach was deltopectoral, with soft-tissue preparation, tenotomy of the subscapularis and tenodesis of the long head of the biceps. The humeral head was resected by a free-hand technique, restoring patient's anatomic version and inclination. The articular centre of rotation was determined using adapted instruments, followed by reaming of an orifice and positioning of a nucleus (three sizes with three fins and plasma-sprayed titanium) and implantation of a humeral head of the desired size (Simpliciti system) (Tornier SAS, Montbonnot, France). A glenoid component was implanted in 40 cases.

The following outcomes were evaluated intra-operatively and post-operatively:

- Primary fixation of the nucleus intra-operatively (satisfactory or unsatisfactory). When it was possible to manualy mobilize the implant, the primary fixation was evaluated as unsatisfactory.

- Functional results in terms of mobility and using the Constant score [9].

- Pre-operative and post-operative radiographic evaluation consisted of true anteroposterior radiographs of the glenohumeral joint (with the humerus in neutral, internal rotation and external rotation) and an axillary view radiograph. All radiographs were made under fluoroscopic control with use of a standardized protocol. Radiographic appearance in frontal and sagittal views at 45 days, three months, six months and last follow-up (standard digital $\mathrm{x}$-rays) with particular attention to periprosthetic

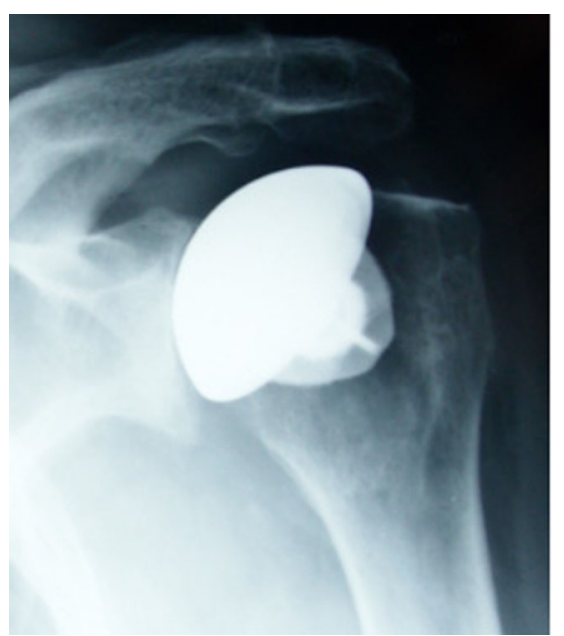

Fig. 1 Revision (6 months after surgery) of a hemiarthroplasty

osteolysis that could lead to implant loosening. The radiographic evaluation protocol was established in advance and all images were assessed by the same observer (PC). We also performed CT scan in order to evaluate bone resorbtion around the prosthesis in some patients. All patients provided informed consent for their participation in this study, which had been approved by the institutional review board in advance (IDRCB 2013AO1788-37).

\section{Statistical analysis}

The Tukey-Krammer multiple comparison post-hoc test was used to assess differences in clinical and radiographic outcomes among the patients with different indications.

\section{Results}

No haematomas, infections, complex regional pain syndromes, nor nervous complications were observed during the follow-



Fig. 2 The nucleus presented very good primary stability with considerable osteointegration and no signs of loosening 
Table 1 Pre and post operative range of motion

\begin{tabular}{llllll}
\hline Mobility & Passive AE & Active AE & Passive ER1 & Active ER1 & Active IR \\
\hline Pre-op & $85^{\circ}\left(50^{\circ} / 150^{\circ}\right)$ & $84^{\circ}\left(30^{\circ} / 120^{\circ}\right)$ & $20^{\circ}\left(-20^{\circ} / 70^{\circ}\right)$ & $15^{\circ}\left(-20^{\circ} / 50^{\circ}\right)$ & 3.3 points $(0 / 10)$ \\
Post op & $141^{\circ}\left(100^{\circ} / 170^{\circ}\right)$ & $131^{\circ}\left(90^{\circ} / 170^{\circ}\right)$ & $20^{\circ}\left(0^{\circ} / 60^{\circ}\right)$ & $15^{\circ}\left(0^{\circ} / 50\right)$ & 6.8 points $(0 / 10)$ \\
\hline
\end{tabular}

up period. At last follow-up, two patients had been re-operated upon. One was a revision (6 months after surgery) of a hemiarthroplasty in a 78 year-old man (primary osteoarthritis), due to residual pain and radiographic signs of periprosthetic osteolysis (Fig. 1). The nucleus presented very good primary stability with considerable osteointegration and no signs of loosening (Fig. 2). A posteriori the patient was diagnosed with glenoditis. Another was a revision (3 years after surgery) of a total shoulder replacement in a 78 years-old woman to a reversed TSA, due to massive anterosuperior tear of the rotator cuff with pseudoparalysis of the shoulder. The nucleus also presented very good primary stability with no signs of loosening or macroscopic osteolysis.

In four women aged 62 to 72 years, the intra-operative assessment of primary fixation of the stemless component was unsatisfactory (insufficient), and the surgeons preferred to implant a classic cemented stemmed prosthesis. In the remaining 43 shoulder implants, the intra-operative assessment of primary fixation was satisfactory (sufficient).

At a mean follow-up of 35 months (range 24-48), the mean anterior flexion was $131^{\circ}$ (mean gain of $48^{\circ}$ ), the mean active external rotation was $15^{\circ}\left(-10\right.$ to $\left.45^{\circ}\right)$ and the mean internal rotation was 4.7 points (out of 10) (Table 1) and the mean Constant score was 69 points (mean gain of 36 points) (Table 2) $(p<0.05)$. Patients operated for post-traumatic arthritis had lower improvement in anterior flexion (mean gain of $26^{\circ}$ ) and Constant score (mean gain of 21 points) $(p<0.05)$

Radiographic assessment revealed periprosthetic radiolucencies as of the sixth post-operative week in 17 cases (Fig. 1) of which only one case was symptomatic and revised as mentioned above. This observation led the authors to collect computed tomography (CT) scans to screen for osteolysis in eight cases. On various slices in three planes, the observers found no evidence of bone loss that could lead to suspicions of early loosening; so we did not request $\mathrm{CT}$ for the 17 patients (Fig. 3).

Table 2 Pre and post operative Constant score

\begin{tabular}{llllll}
\hline Constant score & Pain & Activity & Mobility & Strengh & Global \\
\hline Pre op & $6(3 / 12)$ & $10(6 / 14)$ & $14(8 / 34)$ & $3(0 / 10)$ & $33(18 / 63)$ \\
Post op & $13(8 / 15)$ & $16(12 / 20)$ & $29(2 / 38)$ & $11(3 / 18)$ & $69(48 / 88)$ \\
\hline
\end{tabular}

\section{Discussion}

The results of this prospective clinical series confirm the hypothesis that stemless shoulder arthroplasty does not negatively impact short term outcomes. In four cases, the primary fixation was deemed insufficient. The Constant score was 69 points with a mean gain of 36 points $(p<0.05)$. The group of patients operated for post-traumatic arthritis $(n=12)$ presented inferior gain of mobility (mean gain $26^{\circ}$ ) and Constant score (mean gain 21 points) $(p<0.05)$. Two patients were reoperated and radiolucent lines were observed in 17 other cases.

The main limitation of the present study is that is involves multiple centre and four different surgeons. The mean followup is 35 months with a minimum follow-up of 24 months, which is relatively short for evaluating outcomes of arthroplasty. Moreover, the present study lacks a control group, and is not as conclusive as a randomized controlled trial.

There exist numerous anatomic variations of the proximal humerus [10]. The purpose of shoulder arthroplasty is to reproduce the offset between the articular centre and of the humeral head and the humeral diaphyseal axis. In cases of high anatomic deformity, however, the use of a humeral stem could compromise this goal.

Since the first publication of Neer [1], the design of the humeral stem continued to evolve, and implants available at present are of the fourth generation. Design changes included

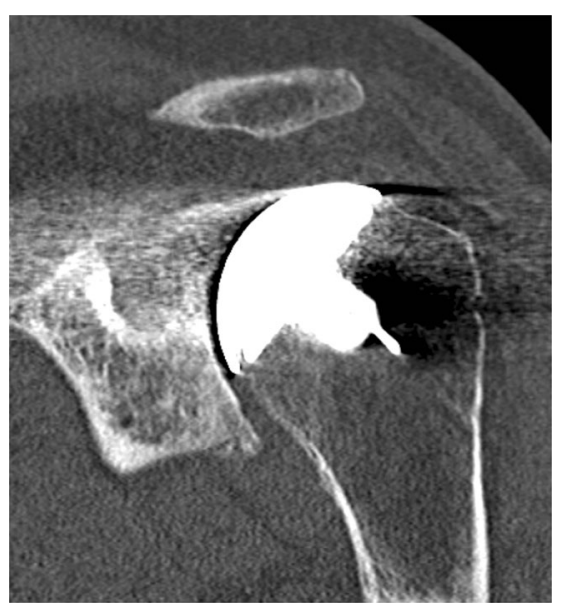

Fig. 3 On various slices in three planes, the observers found no evidence of bone loss that could lead to suspicions of early loosening 


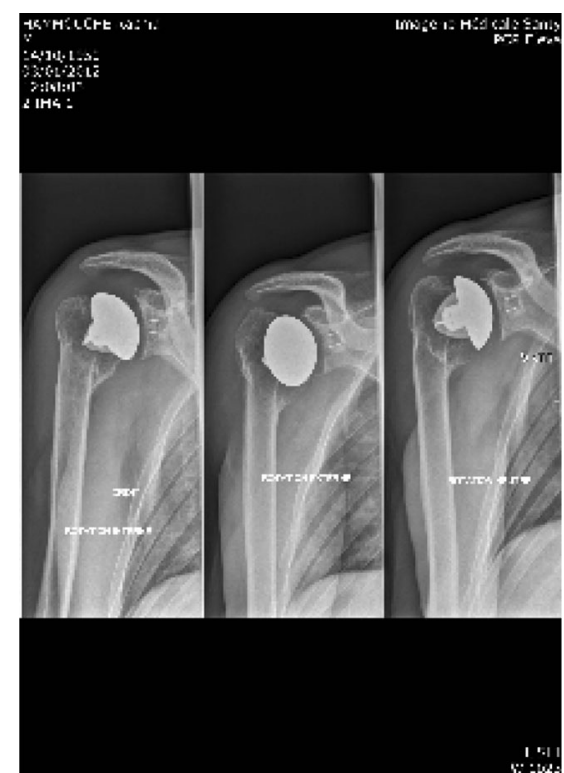

Fig. 4 An example of valgus post-traumatic arthritis with an anatomic reconstruction

reduction or omission of fins, modifications to the shape of the stem, new surface treatments and elimination of cement. Complications related to the humeral stem are well documented, including intra-operative humeral fractures, stress shielding, loosening and post-traumatic fractures. In addition, complications may arise during stem removal during revision due to infection or conversion to a reversed TSA.

In four cases, the primary fixation was deemed insufficient intra-operatively, which confirms the need to provide surgeons with the full set of implants and instruments to implant a stemmed prosthesis if need be.

The concept of stemless shoulder arthroplasty is not recent [11]. Levy and Copeland $[12,13]$ introduced the notion of shoulder resurfacing. The short- and mid-term outcomes are good, though it remains challenging to reproduce normal anatomy, with risks of implant oversizing or varus misalignment. Furthermore, elimination of the humeral resection limits exposure of the glenoid. Resection of the humeral head by 'freehand' technique to reproduce anatomic version favors adequate reconstruction. Lebon et al. [14] recently reported outcomes of shoulder resurfacing compared to those of anatomic stemmed TSA and concluded that stemmed implants produced superior results.
In regards to post-traumatic arthritic with malunion, stemless shoulder implants enable surgeons to perform an arthroplasty even in cases of distortions of the humerus due to fracture misalignments. Figure 4 illustrates an example of valgus post-traumatic arthritis with an anatomic reconstruction. Nevertheless, post-traumatic arthritic with malunion remain difficult to treat. Figure 4 shows how the implantation of a shoulder implant could lead to a rotator cuff lesion, which is why the implant was implanted in varus. Pape et al. [15] reported that results of shoulder arthroplasty after varus nonunion were inferior. In the case of type four humeral sequelae, according to the classification of Boileau et al. [16], the stemless shoulder arthroplasty is not recommended.

Our clinical results in terms of pain (mean gain 7 points), mobility (mean gain $48^{\circ}$ ) and overall satisfaction $(87 \%$ of patients) reflect a significant improvement in Constant score (mean gain 36 points), which is comparable to outcomes of stemmed TSA (17). Two articles reported outcomes of stemless shoulder arthroplasty: Huguet et al. [8] reported results of a series of 72 stemless TSA with minimum follow-up of two years with mean Constant score of 75 points and no signs of loosening. Habermeyer et al. [7] found signs of calcar resorption (Table 3).

Radiographic assessment showed no signs of early migration, nor loosening over time at the longest follow-up of four years for the very first cases. However, periprosthetic radiolucent lines were observed at the upper zones in 17 shoulders. This led us to extend our investigation of early loosening by performing CT scans on eight patients. None of the CT scans revealed signs of loosening (Fig. 3).

Two patients required surgical revision. The first case was revised due to persistent pain and presence of glenoid radiolucencies that led to suspicions of early loosening. During the revision procedure, the nucleus appeared adequately fixed (Fig. 1). A posteriori, the diagnosis was early glenoid wear. The second case was revised for pseudoparalysis of the shoulder due to a massive anterosuperior tear of the rotator cuff. Again, during the revision procedure, the nucleus appeared adequately fixed. Both patients received a reversed TSA and their revision procedure was relatively simple because it did not involve extraction of a humeral stem, hence confirming the supposed benefits of stemless shoulder arthroplasty.
Table 3 Comparison of stemless arthroplasty reports with 2-year minimum results

\begin{tabular}{llllllll}
\hline Author & Implant & $\begin{array}{l}\text { Number } \\
\text { of } \\
\text { patients }\end{array}$ & $\begin{array}{l}\text { years of } \\
\text { minimum } \\
\text { follow up }\end{array}$ & $\begin{array}{l}\text { Hemi or total } \\
\text { arthroplasty }\end{array}$ & $\begin{array}{l}\text { Constant } \\
\text { score }\end{array}$ & $\begin{array}{l}\text { Forward } \\
\text { elevation }\end{array}$ & $\begin{array}{l}\text { external } \\
\text { rotation }\end{array}$ \\
\hline $\begin{array}{c}\text { Huguet et al. } \\
\text { Habermeyer } \\
\text { et al. }\end{array}$ & TESS & 63 & 3 & Both & 75 & $145^{\circ}$ & $40^{\circ}$ \\
\hline
\end{tabular}


Open Access This article is distributed under the terms of the Creative Commons Attribution 4.0 International License (http:// creativecommons.org/licenses/by/4.0/), which permits unrestricted use, distribution, and reproduction in any medium, provided you give appropriate credit to the original author(s) and the source, provide a link to the Creative Commons license, and indicate if changes were made.

\section{References}

1. Neer CS 2nd (1974) Replacement arthroplasty for glenohumeral osteoarthritis. J Bone Joint Surg Am 56(1):1-13

2. Papadonikolakis A, Neradilek MB, Matsen FA 3rd (2006) Failure of the glenoid component in anatomic total shoulder arthroplasty: a systematic review of the English-language literature between 2006 and 2012. J Bone Joint Surg Am 95(24):2205-12

3. Sperling JW, Hawkins RJ, Walch G, Mahoney AP, Zuckerman JD (2013) Complications in total shoulder arthroplasty. Instr Course Lect 62:135-41

4. Gonzalez JF, Alami GB, Baque F, Walch G, Boileau P (2011) Complications of unconstrained shoulder prostheses. J Shoulder Elbow Surg 20(4):666-82

5. Aldinger PR, Raiss P, Rickert M, Loew M (2010) Complications in shoulder arthroplasty: an analysis of 485 cases. Int Orthop 34(4): $517-24$

6. Raiss P, Edwards TB, Deutsch A, Shah A, Bruckner T, Loew M et al (2014) Radiographic changes around humeral components in shoulder arthroplasty. J Bone Joint Surg Am 96(7)

7. Habermeyer P, Lichtenberg S, Tauber M, Magosch P (2015) Midterm results of stemless shoulder arthroplasty: a prospective study. J Shoulder Elbow Surg 24(9):1463-72. doi:10.1016/j. jse.2015.02.023
8. Huguet D, DeClercq G, Rio B, Teissier J, Zipoli B, Group T (2010) Results of a new stemless shoulder prosthesis: radiologic proof of maintained fixation and stability after a minimum of three years follow-up. J Shoulder Elbow Surg 19(6):847-52

9. Constant CR, Murley AH (1987) A clinical method of functional assessment of the shoulder. Clin Orthop Relat Res 214:160-4

10. Boileau P, Walch G (1997) The three-dimensional geometry of the proximal humerus. Implications for surgical technique and prosthetic design. J Bone Joint Surg (Br) 79(5):857-65

11. Churchill RS (2014) Stemless shoulder arthroplasty: current status. J Shoulder Elbow Surg 23(9):1409-14

12. Levy O, Copeland SA (2004) Cementless surface replacement arthroplasty (Copeland CSRA) for osteoarthritis of the shoulder. J Shoulder Elbow Surg 13(3):266-71

13. Levy O, Tsvieli O, Merchant J, Young L, Trimarchi A, Dattani R et al (2015) Surface replacement arthroplasty for glenohumeral arthropathy in patients aged younger than fifty years: results after a minimum ten-year follow-up. J Shoulder Elbow Surg 24(7):1049 60

14. Lebon J, Delclaux S, Bonnevialle N, Rongieres M, Bonnevialle P, Mansat $P$ et al (2014) Stemmed hemiarthroplasty versus resurfacing in primary shoulder osteoarthritis: a single-center retrospective series of 78 patients. Orthop Traumatol Surg Res 100(6 Suppl):S327_ 32

15. Pape G, Tonne L, Raiss P, Loew M, Zeifang F (2013) Prostheses for fractures of the proximal humerus: presentation of current results of anatomical fracture shoulder prosthesis under consideration of alternative concepts]. Orthopade 42(3):164-9

16. Boileau P, Chuinard C, Le Huec JC, Walch G, Trojani C (2006) Proximal humerus fracture sequelae: impact of a new radiographic classification on arthroplasty. Clin Orthop Relat Res 442:121-30 